\title{
煤基炭泡沫孔结构调控
}

\author{
徐国忠 ${ }^{1,2}$, 金文武 ${ }^{1}$, 曾狺榕 ${ }^{2}$, 邹继兆 ${ }^{2}$, 熊信柏 ${ }^{2}$, 黄 麟 $^{2}$, 赵振宁 1
}

(1. 辽宁科技大学化工学院, 辽宁省先进煤焦化技术重点实验室, 鞍山 114051, 2. 深圳大学材料学院, 深圳市特 种功能材料重点实验室, 深圳陶瓷先进技术工程实验室, 深圳 518060)

摘 要: 以肥煤镜质组富集物为前驱体, 采用高压渗氮法制备煤基炭泡沫, 研究了发泡温度、发泡压力和发泡时间 对炭泡沫孔结构的影响。利用 SEM 观察炭泡沫的孔胞形貌, 同时利用 Nano Measurer 分析软件统计 SEM 照片孔 胞直径分布和孔喉直径分布以及平均孔径。结果表明: 微孔塑料成核理论可以定性解释炭泡沫的孔结构变化趋 势。发泡温度的升高导致成核密度增加, 同时导致气体在胶质体的溶解度降低, 不利于孔胞长大。发泡压力的增 大导致炭泡沫的孔胞密度增加，临界成核半径降低，同时加剧了热聚合反应，导致胶质体的粘度增大，不利于孔 胞长大。发泡时间的延长会使热聚合更加充分，影响胶质体粘度，进而影响孔结构。

关 键 词: 炭泡沫; 孔结构; 成核理论; 煤

中图分类号: TQ383 文献标识码: A

\section{Tailoring of Pore Structure of Coal-based Carbon Foam}

\author{
XU Guo-Zhong ${ }^{1,2}$, JIN Wen-Wu ${ }^{1}$, ZENG Xie-Rong ${ }^{2}$, ZOU Ji-Zhao ${ }^{2}$, XIONG Xin-Bai ${ }^{2}$, \\ HUANG Lin ${ }^{2}$, ZHAO Zhen-Ning ${ }^{1}$
}

(1. Liaoning Key Laboratory of Advanced Coal and Coking Technology, College of Chemical Engineering, University of Science and Technology Liaoning, Anshan 114051, China; 2. Shenzhen Key Laboratory of Special Functional Materials \& Shenzhen Engineering Laboratory for Advance Technology of Ceramics, College of Materials Science and Engineering, Shenzhen University, Shenzhen 518060, China)

Abstract: Vitrinite-concentration of fat coal was used as precursor to prepare carbon foams by nitrizing under high pressure. Influences of foaming temperature, pressure and time on the pore structure of carbon foams were investigated, whose prarameters include bulk density, porosity, morphology, pore cell average diameter, and the distribution of pore cell diameter and pore throats. The morphology of pore cell was observed by SEM. The distributions of pore cell diameters and pore throats as well as the mean diameter were calculated using analytical software of Nano Measurer 1.2. Results show that the nucleation of microcellular thermoplastic foam can qualitatively reveal the variable trend of pore cell structure of carbon foams. Rising of the foaming temperature results in nucleation volume density enhancement. Meanwhile, the rising temperature reduces the gas solubility in plastic mass, which is not conducive to the cell growth. Increasing foaming pressure leads to pore cell density enhancement, whereas the critical nucleation radius decrease. In addition, the increased foaming pressure exacerbates thermal polymerization reaction, increasing viscosity of the plastic mass, which is not conducive to the growth of pore cell. Extending foaming time enables thermal polymerization more sufficiently, which influences the viscosity of the plastic mass

收稿日期: 2015-12-14; 收到修改稿日期：2016-01-27

基金项目：国家自然科学基金(51272161, 51202150); 辽宁省教育厅项目(L2013124); 辽宁科技大学专项基金(2012YY03) National Natural Science Foundation of China (51272161, 51202150); Liaoning Provincial Education Department Foundation (L2013124); Special Foundation of University of Science and Technology Liaoning (2012YY03)

作者简介: 徐国忠(1973-), 男, 副教授. E-mail: gz_xu@163.com

通讯作者: 曾䱣榕, 教授. E-mail: zengxier@szu.edu.cn 
and further affects the pore structure.

Key words: carbon foam; pore structure; nucleation theory; coal

炭泡沫是一种具有三维网状结构的多孔轻质炭 材料, 它既具有炭材料的常规性能外, 又具有孔隙 率、导热率、导电率、密度和抗压强度可调控的特 点 ${ }^{[1]}$, 因此炭泡沫常被用作热管理材料、电磁屏蔽、 吸波材料、电极材料、气体吸附剂、催化剂载体和 生物材料等 ${ }^{[2-7]}$ 。依据炭泡沫孔壁的微观结构, 可以 分为石墨化和非石墨化炭泡沫, 并可以通过与金属 和非金属的复合来获得性能优异的结构材料 ${ }^{[8]}$, 因 而炭泡沫的制备及应用研究受到广泛关注。煤基炭 泡沫由于原料来源广泛、制备成本低而最具产业化 前景。1999 年美国西弗吉尼亚大学的 Stiller 教授 ${ }^{[9]}$ 开发了用煤作前驱体制备炭泡沫的技术, 即以沥青 烯为原料制备炭泡沫。但从煤中分离沥青烯过程复 杂, 因此制备成本偏高。为了降低制备成本, Rogers 教授 ${ }^{[10]}$ 以自由膨胀序数为 $3.5 \sim 5$ 的低灰低硫煤为原 料, 采用高压渗氮气法制备炭泡沫。Calvo 等 ${ }^{[11]}$ 利用 具有较好塑性的烟煤为前驱体, 在不同发泡温度和 压力下经两步热处理制备炭泡沫, 孔径随压力升高 而减小, 孔容随温度升高而增加。之后, Calvo 等 ${ }^{[12]}$ 利用不同挥发分的烟煤为原料, 采用高压渗氮气法 制备炭泡沫, 发现随着烟煤流动性提高, 炭泡沫的 体积密度降低。Matviya 等 ${ }^{[13]}$ 以强粘结性、低灰和 低硫的煤为原料, 首先通过振动压实煤颗粒, 然后 在煤床上施加一定压力, 再通过两步加热法制备出 煤基炭泡沫。以上制备煤基炭泡沫的方法均以自由 膨胀序数为 3.5 5 低灰低硫的煤为原料。我国中等 变质程度烟煤的灰分和硫分较高, 而且混煤居多, 不适合制备炭泡沫。为此, 徐国忠等 ${ }^{[14]}$ 采用浮选液 分离富集中等变质程度烟煤的塑性成分(镜质组富 集物), 以其为前驱体, 经高压渗氮法或自生压法制 备出孔径分布均匀且抗压强度较高的炭泡沫。

炭泡沫的性质与孔结构(包括体积密度、气孔 率、孔形貌、孔胞直径分布、孔喉直径分布以及平 均孔胞直径) 密切相关, 而孔结构主要受前驱体性 质和制备工艺条件的影响, 因此, 炭泡沫的孔结构 裁剪是炭泡沫性能优化的核心 ${ }^{[15]}$ 。迄今为止, 系统 研究对煤基炭泡沫的孔结构影响的关键因素鲜有报 道。为此, 本工作选用肥煤镜质组富集物为前驱体, 利用高压渗氮法制备炭泡沫。系统研究原料的捣固 压力、发泡压力、发泡温度和恒温时间对于炭泡沫 孔结构的影响规律, 并尝试利用微孔塑料成核理论
对炭泡沫的孔结构变化进行解释。

\section{1 实验方法}

\section{1 前驱体}

将干燥的肥煤粉碎成 $\phi 180 \sim 250 \mu \mathrm{m}$ 的煤粉。以 密度为 $1.35 \mathrm{~g} / \mathrm{cm}^{3}$ 的氯化锌溶液作为比重液, 采用 重力浮选法进行分离, 取出上层物进行抽滤、洗 涤、干燥得到肥煤镜质组富集物, 作为制备炭泡沫 的前驱体。肥煤及其镜质组的工业分析和元素分析 如表 1 所示, 可以看出, 相对肥煤而言, 镜质组富 集物的灰分由 $8.52 \%$ 降到 $3.5 \%$, 而挥发分提高了 $5.51 \%$; 元素分结果表明, 碳元素含量降低, 而其他 元素含量均有不同程度提高。根据 ASTMD2639-98 测定前驱体的基氏流动度。采用热重分析仪 (Diamond TG/DTA6300)分析镜质组富集物热失重 情况, 量取样品 15 17 mg, 控制 $\mathrm{N}_{2}$ 流速为 $100 \mathrm{~mL} / \mathrm{min}$, 然后以 $2^{\circ} \mathrm{C} / \mathrm{min}$ 升至 $1000^{\circ} \mathrm{C}$ 。

图 1 和图 2 分别为前驱体的热重和基氏流动度 曲线。由图 1 知, 温度上升到 $465^{\circ} \mathrm{C}$ 时前驱体的失重 率最大。由图 2 发现, 基氏流动度随着温度的升高 呈现先升高再降低的变化趋势。镜质组富集物的开 始软化温度为 $371^{\circ} \mathrm{C}$ 、固化温度为 $483^{\circ} \mathrm{C}$ 、最大基氏 流动度对应温度为 $433^{\circ} \mathrm{C}$ 、塑性温度区间为 $112^{\circ} \mathrm{C}$ 。 对该曲线进行非线性拟合，发现基氏流动度随温度 变化近似呈现高斯分布，相关系数 $\left(R^{2}\right)$ 为 $95 \%$ 。镜质 组富集物的基氏流动度和 $\mathrm{TG}$ 数据分析为发泡温度 的选择提供了基础, 即发泡温度必须在软化温度和 固化温度之间选取。

\section{2 炭泡沫制备}

将 $70 \mathrm{~g}$ 肥煤镜质组富集物放入金属模具中, 利 用压力机施加一定压力。然后置于高压反应釜中, 以 $2^{\circ} \mathrm{C} / \mathrm{min}$ 升温至软化点温度, 充入氮气达到要求 压力, 再继续以 $2^{\circ} \mathrm{C} / \mathrm{min}$ 升温至最终发泡温度, 恒

表 1 肥煤及其镜质组富集物的工业分析和元素分析结果 Table 1 Proximate analysis and ultimate analysis of the fat coal and its vitrinite-concentration

\begin{tabular}{|c|c|c|c|c|c|c|c|c|}
\hline \multirow{2}{*}{$\begin{array}{c}\text { precur- } \\
\text { sors }\end{array}$} & \multicolumn{3}{|c|}{ Proximate analysis $/ \mathrm{wt} \%$} & \multicolumn{5}{|c|}{ Ultimate anasysis/wt\% } \\
\hline & $M_{\mathrm{ad}}$ & $A_{\mathrm{d}}$ & $V_{\mathrm{daf}}$ & $\mathrm{C}$ & $\mathrm{H}$ & $\mathrm{O}$ & $\mathrm{N}$ & $\mathrm{S}$ \\
\hline & 1.6 & 85 & 38.29 & 8 & 1.02 & I. & 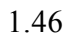 & 0.62 \\
\hline Vitrinite & 1.7 & 3.50 & 43.80 & 86.45 & 5.73 & 5.65 & 1.53 & 0.64 \\
\hline
\end{tabular}




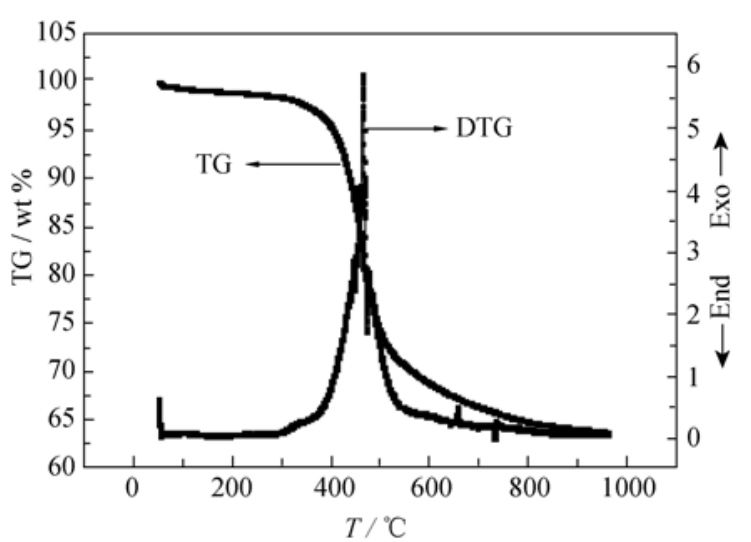

图 1 前驱体的 TG 和 DTG 曲线

Fig. 1 TG and DTG curves of the precursor

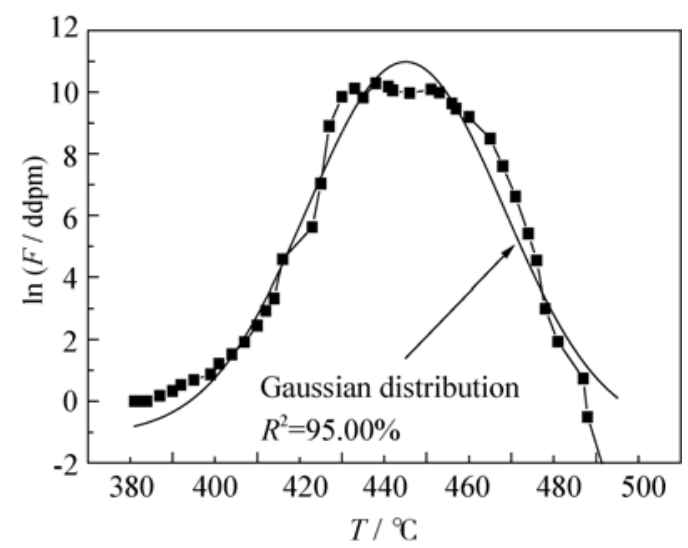

图 2 前驱体基氏流动度自然对数与温度的关系

Fig. 2 Correlation between LNF and temperature

温一定时间 (定义为发泡时间)。恒温结束后, 以 $2 \mathrm{MPa} / \mathrm{min}$ 的速度放压至常压, 并关闭出气阀。继续以 $2^{\circ} \mathrm{C} / \mathrm{min}$ 升温至 $550^{\circ} \mathrm{C}$, 恒温 $20 \mathrm{~min}$, 自然降温至室温, 得到炭泡沫初生体。炭泡沫制备装置示意图如图 3 所 示。将炭泡沫初生体转移到管式炉中进行炭化处理, 在 高纯氮气的保护下, 以 $2^{\circ} \mathrm{C} / \mathrm{min}$ 升温至 $1000^{\circ} \mathrm{C}$, 恒温 $120 \mathrm{~min}$, 然后自然降温至室温, 得到煤基炭泡沫。

\section{3 炭泡沫的表征}

(1) 炭泡沫的体密度和气孔率

将炭泡沫研磨至粒度小于 $0.2 \mathrm{~mm}$, 然后采用比 重瓶法(GB/T 217 21996)测试其真密度 $\left(\rho_{\mathrm{T}}\right)$ 。将炭泡 沫加工成尺寸为 $20 \mathrm{~mm} \times 20 \mathrm{~mm} \times 10 \mathrm{~mm}$ 的长方体, 利用卡尺精确测量试样的长 $(a)$ 、宽 $(b)$ 和高 $(c)$, 称量 试样的质量 $(M)$, 按公式 (1) 计算试样的体积密 度 $\left(\rho_{\mathrm{V}}\right)$, 按公式 $(2)$ 计算试样的气孔率 $(P)$ 。

$$
\begin{gathered}
\rho_{\mathrm{V}}=\frac{M}{a b c} \\
P=1-\frac{\rho_{\mathrm{V}}}{\rho_{\mathrm{T}}}
\end{gathered}
$$

（2）炭泡沫的孔胞形貌和孔径分布

采用 JSM-6480LV 型扫描电子显微镜观察炭泡

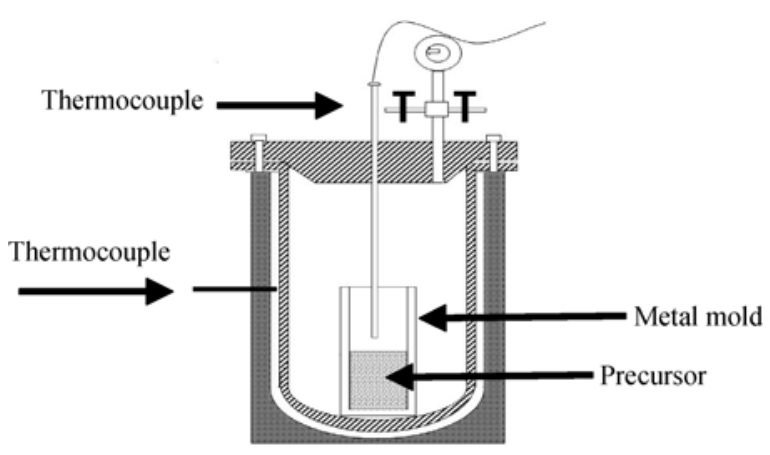

图 3 碳泡沫制备装置示意图

Fig. 3 Schematic diagram of the oxperimental set-up

沫的形貌，并通过Nano Measurer 1.2粒径分析软件 分别统计SEM照片孔胞直径分布、平均孔径以及孔 喉直径分布。

\section{2 结果与讨论}

\section{1 微孔塑料和炭泡沫发泡过程}

煤在一定发泡温度、压力和时间下的发泡过程 与微孔塑料的发泡过程相似。即随着温度升高, 前 驱体(塑料或镜质组富集物)经过下述几个过程转化 为泡沫: (1)前驱体受热软化熔融产生熔体; (2)气体 遵循亨利定律溶于熔体; (3)泄压使熔体中的气体达 到过饱和而成核，即胞核形成; (4)孔胞长大; (5)降 温孔胞固化, 形成微孔塑料或炭泡沫初生体; (6)炭 泡沫初生体继续升温炭化得到炭泡沫。但二者也存 在不同之处: 在步骤(1)中塑料产生熔体是物理过程, 而镜质组富集物产生熔体过程中，存在热分解和热 聚合反应; 在步骤(2)中, 溶于塑料形成的熔体中的 气体为外加气体(氮气或 $\mathrm{CO}_{2}$ 气体), 而溶于镜质组 富集物形成熔体中的气体, 既包括外加气体也包括 煤热分解产生的气体。基于上述分析，微孔塑料和 炭泡沫的发泡过程存在相似之处。

根据 Colton 和 Suh 的微孔塑料经典均相成核理 论, 胞核生成需要克服的能垒、临界成核半径以及 成核速率分别见公式(3 6) ${ }^{[16]}$ 。由式(3)可知, $\Delta p$ 和 $\gamma$ 是影响均相成核临界自由能的两个主要参数, 增大 $\Delta p$ 和减小 $\gamma$ 均会使自由能的变化量降低，进而提高 气泡核的成核密度 ${ }^{[16-17]}$ 。而成核后气泡的形成和长 大则依赖于三个条件, 即化学势条件、力学条件和 流动条件 ${ }^{[18]}$ 。气泡核形成后，气体分子持续从熔体 中扩散进入胞核使其膨胀长大 ${ }^{[18]}$ 。

$$
\Delta G_{\mathrm{homo}}^{*}=\frac{16 \pi \gamma^{3}}{3 \Delta p^{2}}
$$




$$
\begin{gathered}
r^{*}=\frac{2 \gamma}{\Delta p} \\
N_{\text {homo }}=f_{0} c_{0} \exp \left(-\Delta G_{\text {homo }}^{*} / k T\right) \\
r=\frac{2 \gamma}{p}
\end{gathered}
$$

式中: $\Delta G_{\mathrm{homo}}^{*}$ 一成核需要克服的能垒; $\gamma$ 一胶质体与 气泡之间的界面张力; $r^{*}$ 一孔胞的临界半径; $\Delta p$ 一饱 和压力与系统压力(泄压后的系统压力环境压力)差; $N_{\text {homo }}$ 一单位时间单位体积内气泡成核数; $f_{0}$ 一气体 分子进入到临界半径气泡核的速率因子; $C_{0}$ 一气体 分子的浓度; $k$-Boltmann 常数; $T$ 一绝对温度; $p$ 孔胞内气体压力。

\section{2 发泡参数对炭泡沫孔结构影响}

\subsection{1 捣固压力}

在发泡温度、压力和时间分别为 $453^{\circ} \mathrm{C} 、 6 \mathrm{MPa}$ 和 $2 \mathrm{~h}$ 时, 研究捣固压力对炭泡沫的体密度、气孔率 和孔结构的影响规律。如图 4 所示, 随着捣固压力的 升高, 炭泡沫的体密度先降后升, 而气孔率则先升 后降。在捣固压力为 $6 \mathrm{MPa}$ 时, 炭泡沫的体密度取得 最小值为 $0.47 \mathrm{~g} / \mathrm{cm}^{3}$ ，气孔率取得最大值为 $73.33 \%$ 。

图 5 为不同捣固压力制备的炭泡沫的微观形 貌。随着捣固压力的增大, 炭泡沫的孔胞数量增加, 平均孔径逐渐减小。由图 6(a)和(b)可以看出，随捣 固压力的增大，孔胞直径分布和孔喉(孔胞上的窗 口)分布均越来越集中，而且分布峰均向小孔径范 围移动; 炭泡沫孔胞的平均直径减小 (见图 6(c)), 当 捣固压力大于 $10 \mathrm{MPa}$ 时, 炭泡沫的平均孔径变化 不大。前驱体在发泡之前, 施加一定机械压力，目的 是调整前驱体颗粒之间的间隙。每个颗粒受热熔融 形成熔体(煤化工领域俗称胶质体)，当释放压力时 胶质体气相成核长大。核长大除了取决于发泡条件 外，在一定程度上还取决于核长大所需的空间(即

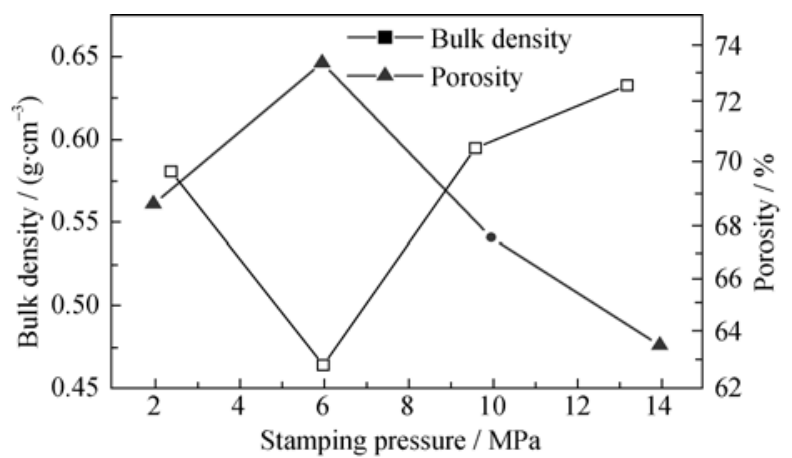

图 4 捣固压力对炭泡沫体密度和气孔率的影响

Fig. 4 Effect of the stamping pressure on the bulk density and porosity of carbon foams
前驱体颗粒周围的间隙)。因此，随着捣固压力提高， 炭泡沫的孔胞直径减小 (见图 5 和图 6(c)), 当捣固压 力大于 $10 \mathrm{MPa}$ 后, 孔径变化不大。

\subsection{2 发泡温度}

当捣固压力、发泡时间和发泡压力分别为 $6 \mathrm{MPa} 、 2 \mathrm{~h}$ 和 $6 \mathrm{MPa}$ 时, 研究发泡温度对炭泡沫的
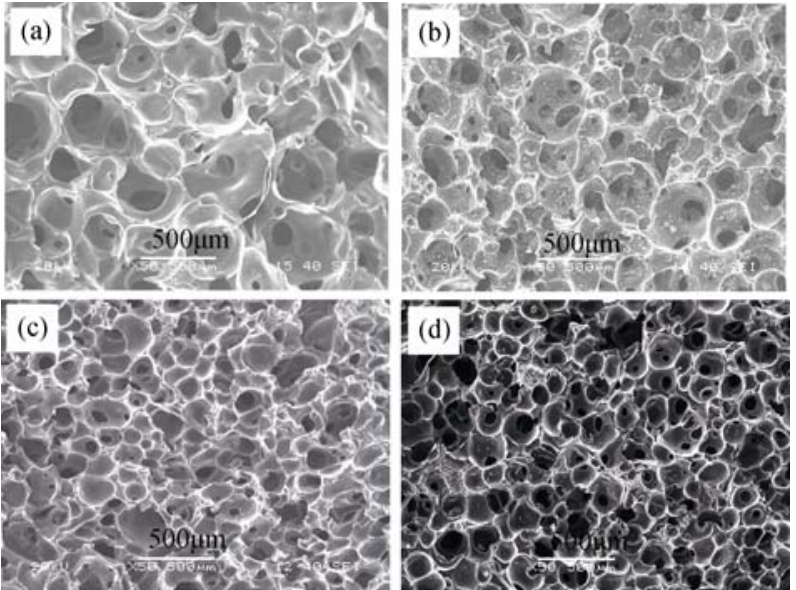

图 5 捣固压力对炭泡沫孔泡形貌的影响

Fig. 5 Effect of stamping pressure on the pore cell morphology of carbon foams

(a) $2 \mathrm{MPa}$; (b) $6 \mathrm{MPa}$; (c) $10 \mathrm{MPa}$; (d) $14 \mathrm{MPa}$
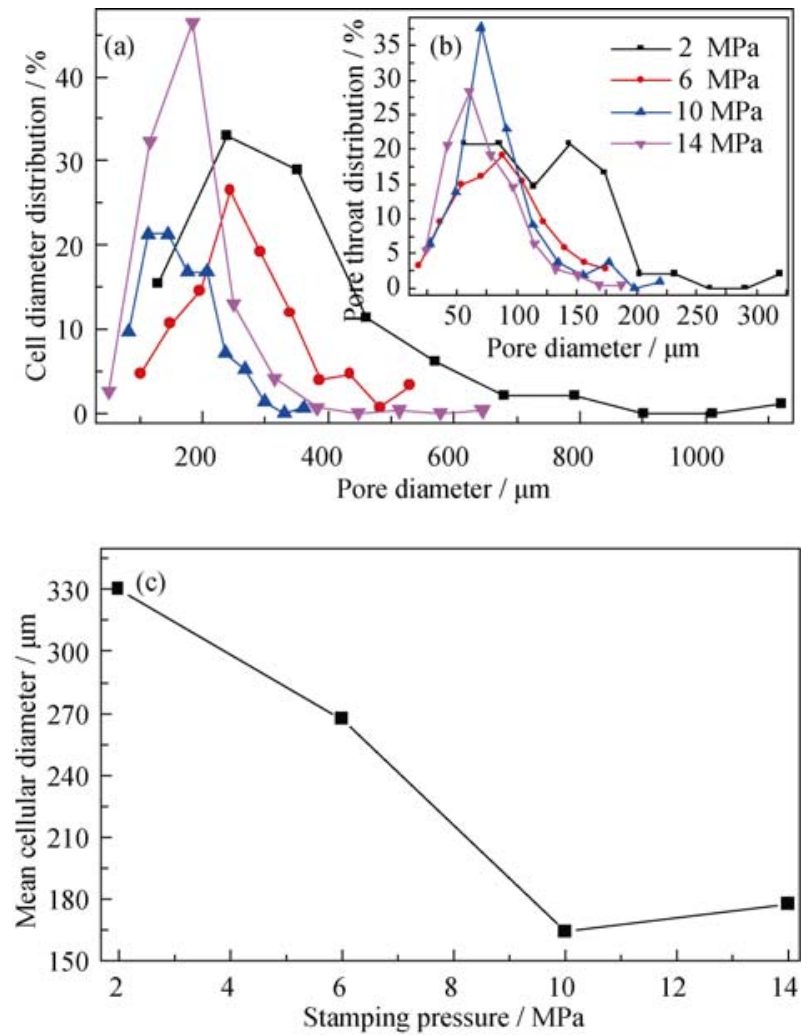

图 6 捣固压力对炭泡沫孔胞直径分布(a)、孔喉直径分布(b) 及平均孔胞直径(c)的影响

Fig. 6 Influence of stamping pressure on pore cell diameter distribution (a), pore throat distribution (b) and mean cell diameter (c) 
孔结构的影响。由图 7 可知, 随着发泡温度的升高, 炭泡沫的体密度先降后升, 而气孔率先升后降。当 发泡温度为 $453^{\circ} \mathrm{C}$ 时, 炭泡沫的体密度获得最小值 为 $0.47 \mathrm{~g} / \mathrm{cm}^{3}$, 气孔率获得最大值为 $73.33 \%$ 。

由微孔塑料均相成核理论, 温度升高成核密 度增大(见公式(5)), 表现出孔胞数量增多, 与图 8 所示结果一致。较低发泡温度 $\left(393^{\circ} \mathrm{C}\right.$ 和 $\left.413^{\circ} \mathrm{C}\right)$ 时, 仍有未发泡的区域存在(见 8(a)和 8(b)), 即成核密度 低所致。文献[19]报道, 随着发泡温度升高, 气体在 熔体中的溶解度降低, 在泄压发生相分离时, 胶质 体内没有充足气体向孔胞内扩散, 推动孔胞长大。 实验结果表明, 发泡温度从 $413^{\circ} \mathrm{C}$ 升至 $473^{\circ} \mathrm{C}$, 得到 炭泡沫的平均直径降低(见图 9(c)), 这一结果与理 论分析一致。从图 2 可以看到, $393^{\circ} \mathrm{C}$ 时对应镜质组 形成胶质体粘度非常高, 不利于孔胞长大, 因此该 温度不适合制备炭泡沫。

\subsection{3 发泡压力}

当捣固压力、发泡温度和时间分别为 $6 \mathrm{MPa}$ 、 $453^{\circ} \mathrm{C}$ 和 $2 \mathrm{~h}$ 时, 研究发泡压力对炭泡沫的孔结构
的影响。由图 10 所示可知，随着发泡压力的提高, 炭泡沫的体密度先降后升, 而气孔率先升后降。当 发泡压力为 $4 \mathrm{MPa}$ 时, 炭泡沫的体密度获得最小 值为 $0.29 \mathrm{~g} / \mathrm{cm}^{3}$, 而气孔率获得最大值为 $83.87 \%$ 。

图 11 和图 12 分别为压力对炭泡沫形貌和孔结构的 影响。

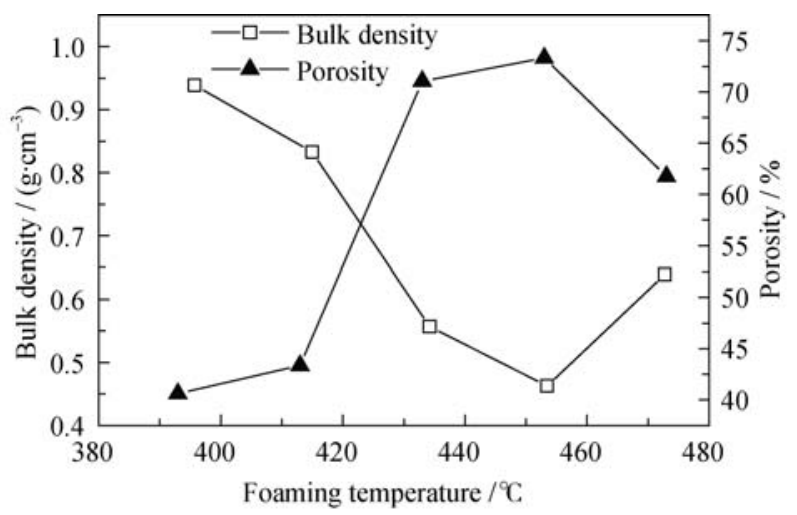

图 7 发泡温度对炭泡沫体密度和气孔率的影响

Fig. 7 Effect of foaming temperature on the bulk density and porosity of carbon foams
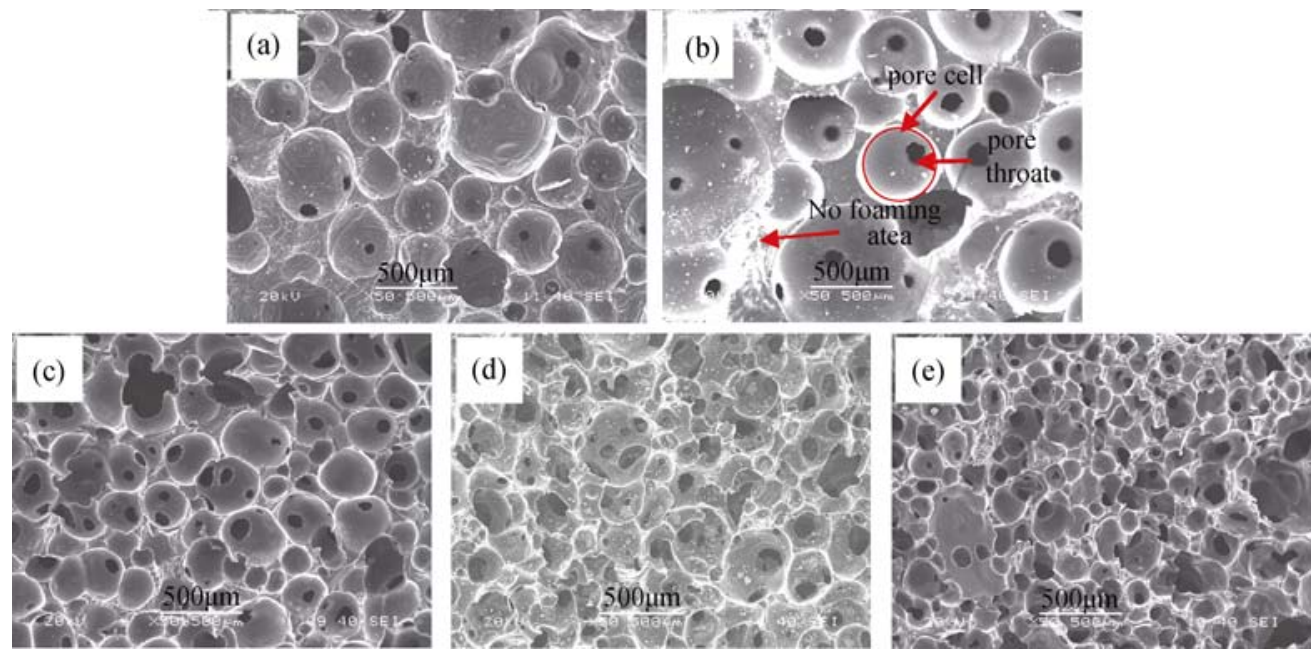

图 8 不同发泡温度下炭泡沫的孔胞 SEM 照片

Fig. 8 Influence of foaming temperature on the pore cell morphology of carbon foams (a) $393^{\circ} \mathrm{C}$; (b) $413^{\circ} \mathrm{C}$; (c) $433^{\circ} \mathrm{C}$; (d) $453^{\circ} \mathrm{C}$; (e) $473^{\circ} \mathrm{C}$
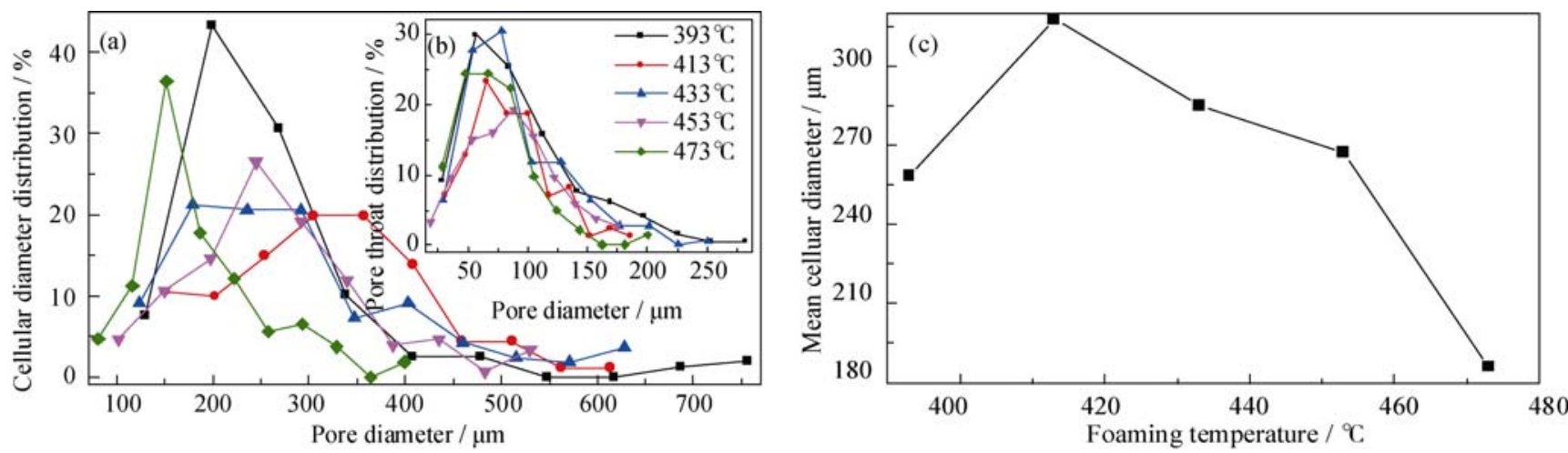

图 9 发泡温度对炭泡沫孔胞直径分布(a)、孔喉直径分布(b)及孔胞的平均直径(c)的影响

Fig. 9 Influence of foaming temperature on pore cell diameter distribution (a), pore throat distribution (b) and mean cell diameter (c) of carbon foams 


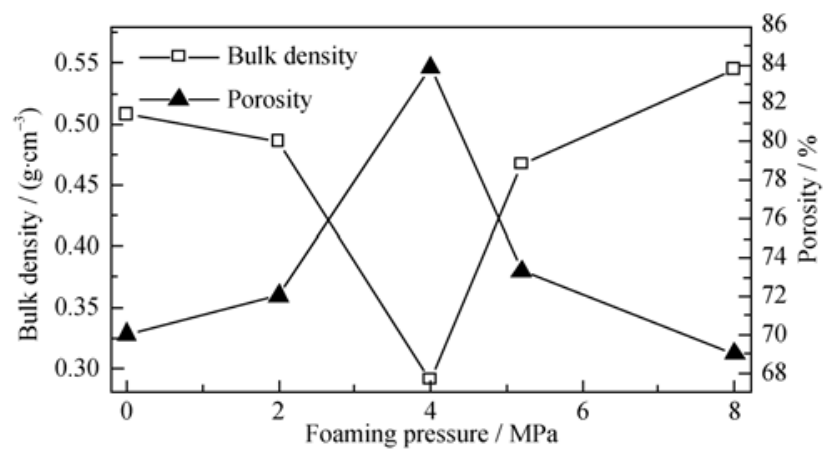

图 10 发泡压力对炭泡沫体密度和气孔率的影响

Fig. 10 Effect of foaming pressure on the bulk density and porosity of carbon foams

根据微孔塑料均相成核理论, 当发泡温度不变 时, 发泡压力越大, 则氮气在胶质体中的溶解度越 高, 导致胶质体中气体的饱和压力越大。泄压后饱 和压力与系统压力 (试验中泄压后的系统压力为环 境压力)差越大, 由公式(3)和(4)可知, 临界成核的
能垒越低，临界成核的半径越小。进而根据公式(5) 可知，成核密度越高，表现出孔胞数量多且分布集 中。与图 11 和图 12(a)显示的结果一致。另外, 发 泡压力的提高有利于热聚合反应进行，因此提高发 泡压力将导致前驱体的平均分子量提高, 进而提高 胶质体的粘度，增加孔胞长大的阻力。由图 12(c)可 知, 发泡压力与平均孔径具有较好负相关性, 与理 论分析一致。

\subsection{4 发泡时间}

当捣固压力、发泡温度和压力分别为 $6 \mathrm{MPa}$ $453^{\circ} \mathrm{C}$ 和 $6 \mathrm{MPa}$ 时, 研究发泡时间对炭泡沫的孔结 构的影响。由图 13 可知，随着发泡时间的延长，炭 泡沫的体密度先降后升，而气孔率先升后降。当发 泡压力为 $2 \mathrm{~h}$ 时, 炭泡沫的体密度取得最小值为 $0.47 \mathrm{~g} / \mathrm{cm}^{3}$ ，气孔率取得最大值为 $73.33 \%$ 。

图 14 和图 15 分别为发泡时间对炭泡沫的孔胞 形貌和孔结构的影响。由图 14 可以直观看到，随着
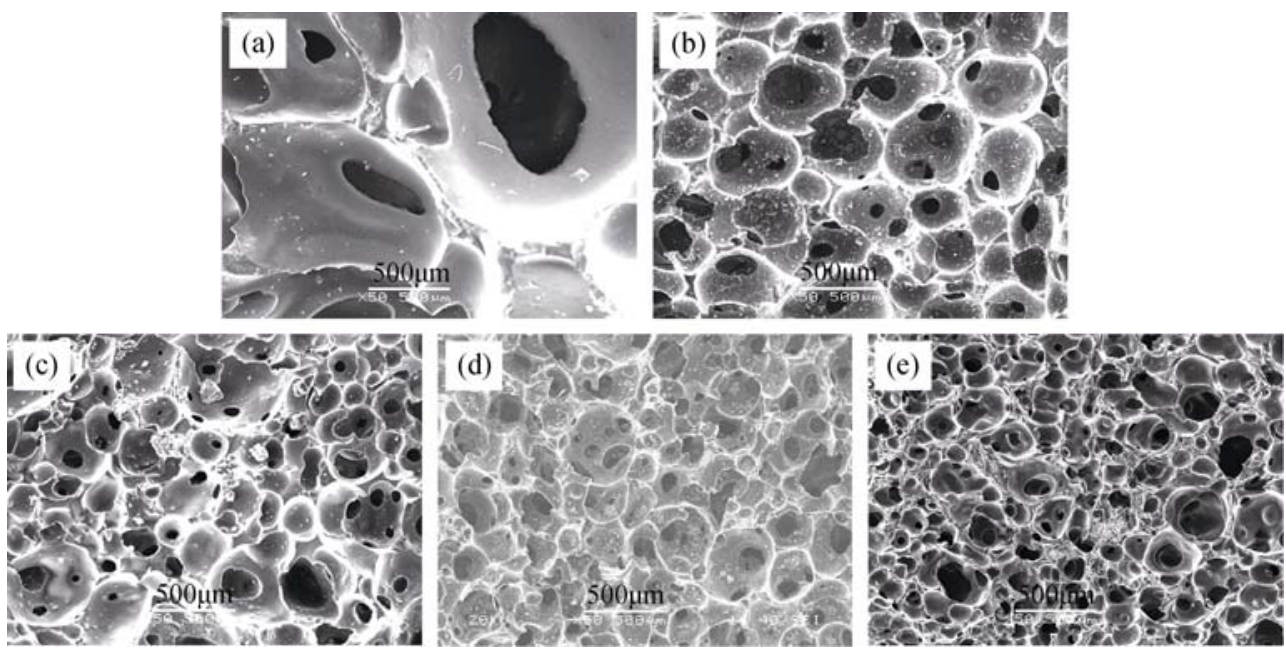

图 11 不同发泡压力下炭泡沫孔胞的 SEM 照片

Fig. 11 Effect of foaming pressure on pore cell morphology of carbon foams (a) $0 \mathrm{MPa}$; (b) $2 \mathrm{MPa}$; (c) $4 \mathrm{MPa}$; (d) $6 \mathrm{MPa}$; (e) $8 \mathrm{MPa}$
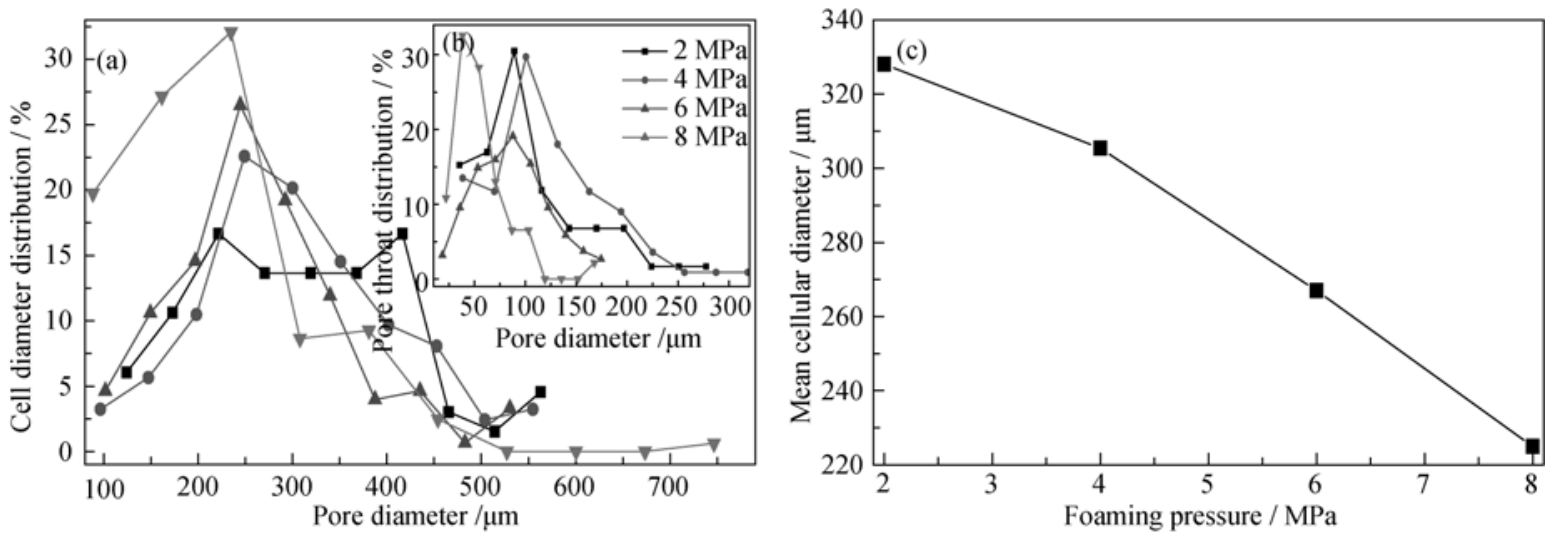

图 12 发泡压力对炭泡沫孔胞直径分布(a)、孔喉直径分布(b)及平均孔胞直径(c)的影响

Fig. 12 Effect of foaming pressure on pore cell diameter distribution (a), pore throat distribution diameter (b) and mean cell diameter (c) of carbon foams 


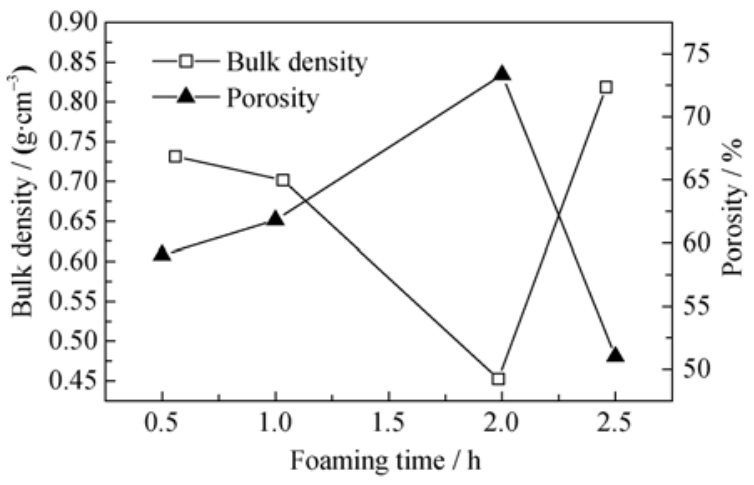

图 13 发泡时间对炭泡沫体密度和气孔率的影响

Fig. 13 Effect of foaming time on the bulk density and porosity of carbon foams
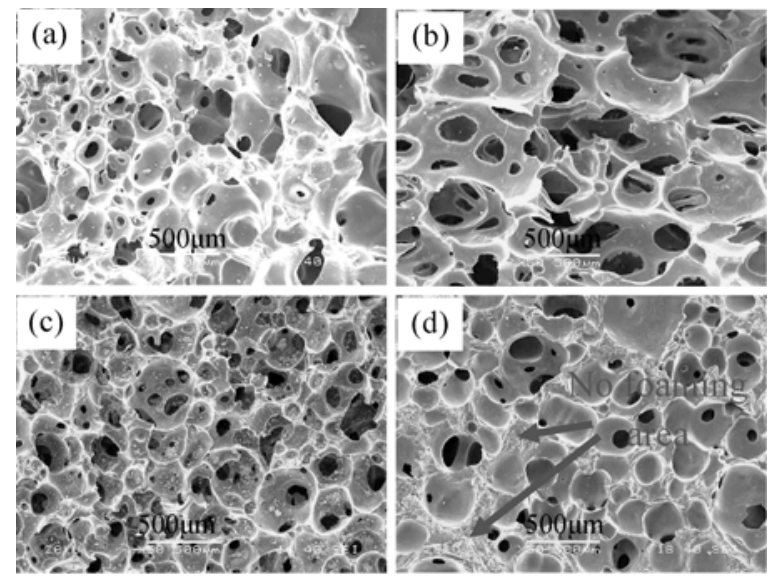

图 14 不同发泡时间的炭泡沫孔胞 SEM 照片

Fig. 14 Influence of the foaming time on cell morphology of carbon foams

(a) $0.5 \mathrm{~h}$; (b) $1 \mathrm{~h}$; (c) $2 \mathrm{~h}$; (a) $2.5 \mathrm{~h}$

发泡时间的延长, 孔胞直径先增大后减小, 发泡时 间为 $1 \mathrm{~h}$, 炭泡沫孔胞直径最大。SEM 照片显示结果 与孔胞平均直径一致(见图 15(c))。由图 15(a)可以看 出, 除了恒温 $1 \mathrm{~h}$ 得到炭泡沫外, 其余炭泡沫的孔胞 直径分布均较集中, 主要集中在 $400 \mu \mathrm{m}$ 以内。孔喉 主要分布在 $200 \mu \mathrm{m}$ 以内, 但恒温 $1 \mathrm{~h}$ 得到炭泡沫的 孔喉分布较宽泛。在发泡温度和压力固定的前提下, 发泡时间越长, 则胶质体的热聚合反应进行得越充 分，胶质体的粘度会增大，进而导致表面张力增大， 由公式(3)可知成核能垒提高, 又由公式(5)可以看出 成核密度降低。图 14(d)的 SEM 照片中存在明显未发 泡的区域证实了上述推断。胶质体粘度高不利于孔胞 的长大, 这是发泡时间延长得到炭泡沫孔胞直径分布 集中(见图 15(a))的内在原因。而炭泡沫平均孔径却随 着发泡时间的延长先增后减(见图 15(c))。这一现象说 明, 存在适合发泡的胶质体粘度和表面张力, 而合适 的粘度和表面张力可以通过发泡时间的调节来实现。
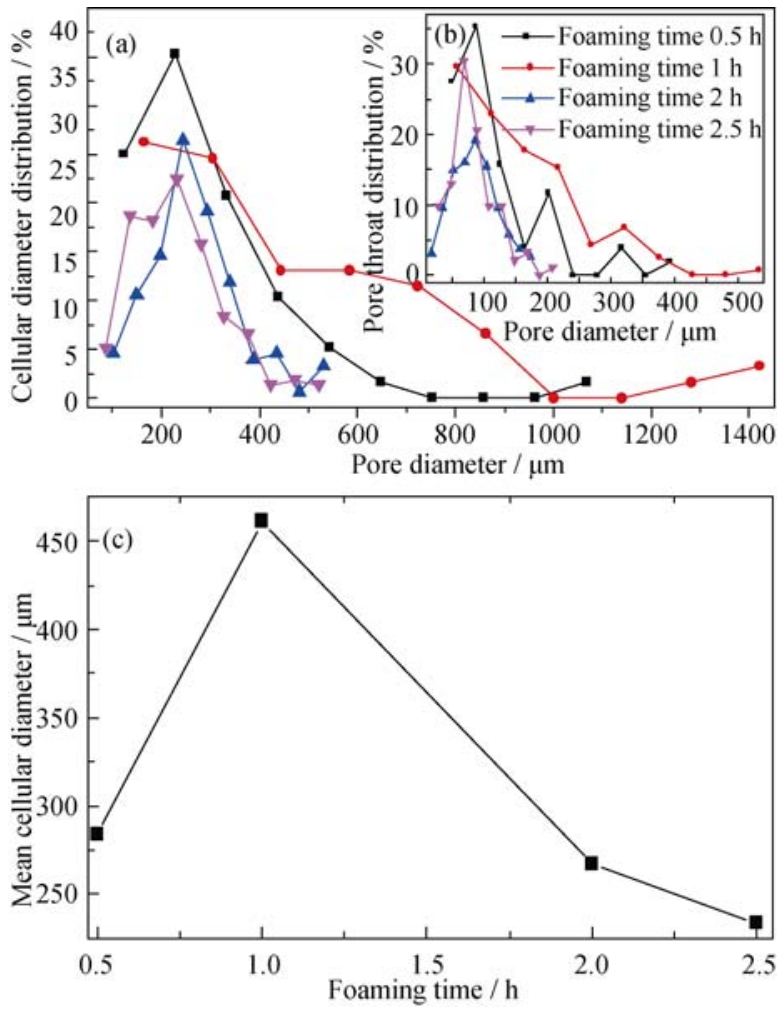

图 15 发泡时间对炭泡沫孔胞直径分布(a)、孔喉直径分布(b) 和孔胞平均直径(c)的影响

Fig. 15 Effect of foaming time on pore cell diameter distribution (a), pore throat distribution (b) and mean cell diameter (c) of carbon foams

\section{3 结论}

1) 实验结果表明, 微孔塑料的成核理论对煤 基炭泡沫的发泡成核行为具有指导意义。

2) 在前驱体的塑性温度区间内，随着发泡温 度的升高, 炭泡沫的孔胞密度逐渐增大, 且孔胞的 平均直径逐渐减小。

3) 随着发泡压力的提高, 炭泡沫的孔胞密度 逐渐增大, 孔胞平均直径逐渐减小。

4) 随着发泡时间的延长, 炭泡沫的孔胞平均 直径呈现先升后降趋势。

5) 捣固压力对炭泡沫的孔结构也具有一定的 影响。随着捣固压力的提高, 炭泡沫的孔胞密度逐 渐增大, 孔胞直径减小。

\section{参考文献:}

[1] MICHIO INAGAKI, QIU JIESHAN, GUO QUANGUI. Carbon foam: Preparation and application. Carbon, 2015, 87: 128-152.

[2] KLETT JAMES, HARDY ROMMIE, ROMINE ERNIE, et al. High-thermal-conductivity, mesophase-pitch-derived carbon foams: effect of precursor on structure and properties. Carbon, 2000, 
38(7): 953-973.

[3] BAO YING, WANG CHUN-XIAO, LING II-CHENG, et al. Effect mechanisms of carbon nanotubes on the supercritical foaming behaviors and mechanical performance of carbon foam. Journal of Inorganic Materials, 2011, 26(10): 1020-1024.

[4] KUMAR R, SINGH A P, CHAND M.et al. Improved microwave absorption in lightweight resin-based carbon foam by decorating with magnetic and dielectric nanoparticles. RSC ADV., 2014, (4): 23476.

[5] GAllego N C, KLETT J W. Carbon foams for thermal management. Carbon, 2003, 41(7): 1461-1466.

[6] NORAKI SANO, HIROSHI AKAZAWA, TAKEYUKI KIKUCHI, et al. Separated synthesis of iron-included carbon nanocapsules and nanotubes by pyrolysis of ferrocene in pure hydrogen. Carbon, 2003, 41(11): 2159-2179.

[7] BRUNETON E, TALLARON C, GRASS-NAULIN N, et al. Evolution of the structure and mechanical behaviour of a carbon foam at very high temperatures. Carbon, 2002, 40(11): 1919-1927.

[8] CHEN FEN, ZHANG HONG-BO, XIONG XIANG, et al. Microstructure and properties of carbon foams reinforced by CVD Pc. Journal of Inorganic Materials, 2008, 26(6): 1184-1188.

[9] STILLER ALFRED H, STANSHERRY PETER G, ZONDLO JOHN W. Method of making a Carbon Foam Material and Resultant Product. US, 5888469A. 1999-3-30.
[10] ROGERS D K. Cellular Coal Products and Pocesses. US, 7588608 2009-09-15.

[11] CALVO MONTSERRAT, GARCIA ROBERTO. Carbon foams from coals. A preliminary study. Fuel, 2005, 84(17): 2184-2189.

[12] CALVO MONTSERRAT, GARCIA ROBERTO, SABINO R MOINELO. Carbon foams from different coals. Energy \& Fuels, 2008, 22(5): 3376-3383.

[13] MATVIYA T M, ROCKS M P A. Production of Carbon Foam from Coals. US, 7767183. 2010-08-03.

[14] 徐国忠, 钟祥云, 赵振宁, 等. 利用预处理烟煤制备煤基炭泡沫 的方法. 中国, C, ZL 201210364642.6, 2014-07-16.

[15] BOGUMILA NAGEL, SLAWOMIRA PUSZ, BARBARA TRZEBICKA. Review: tailoring the properties of macroporous carbon foams. J Mater Sci., 2014, 49: 1-17.

[16] COLTON J S, SUH N P. Nucleation of microcellular thermoplastic foam with additives part I: Theoretical considerations. Polymer Engineering and Science, 1987, 27(7): 485-492.

[17] 吴舜英, 徐敬一. 泡沫塑料成型, 2 版. 北京: 化学工业出版社, 1999: 1-90.

[18] 徐 晓. 对塑料发泡中应用的经典成核理论. 广州: 华南理工大 学博士学位论文, 2000: 14-23.

[19] LEE D, HANSEN V, WALLACE MCCARLIE. From foam rubber to volcanoes: the physical chemistry of foam formation. Journal of Chemical Education, 2004, 81(11): 1581-1583. 